\title{
On the implicit immersed boundary method in coupled discrete element and lattice Boltzmann method
}

\author{
Min Wang ${ }^{1}$, Y.T. Feng ${ }^{2}$, T.M. $\mathrm{Qu}^{2}$
}

1 T-3 Fluid Dynamics and Solid Mechanics Group, Theoretical Division, Los Alamos National Laboratory, Los Alamos, New Mexico 87545, USA

2 Zienkiewicz Centre for Computational Engineering, College of Engineering, Swansea University, Swansea, Wales SA1 8EP, UK

\begin{abstract}
The coupled discrete element method and lattice Boltzmann method (DEMLBM) has increasingly drawn attention of researchers in geomechanics due to its mesoscopic nature since 2000. Immersed boundary method (IBM) and immersed moving boundary (IMB) are two popular schemes for coupling fluid-particle in DEMLBM. This work aims at coupling DEM and LBM using the latest IBM algorithm and investigating its accuracy, computational efficiency and applicability. Two benchmark tests, interstitial fluid flow in an ideal packing and single particle sedimentation in viscous fluid, are carried out to demonstrate the accuracy of IBM through semi-empirical Ergun equation, finite element method (FEM) and IMB. Then, simulations of particle migration with relatively large velocity in Poiseuille flow are utilized to address limitations of IBM in DEMLBM modeling. In addition, advantages and deficiencies of IBM are discussed and compared with IMB. It is found that the accuracy of IBM can be only guaranteed when sufficient boundary points are used and it is not suitable for geomechanical problems involving large fluid or particle velocity.
\end{abstract}

\section{Keywords}

Immersed Boundary Method; Discrete Element Method; Lattice Boltzmann Method; Fluid-Solid Interaction; Immersed Moving Boundary

\section{Introduction}

The fluid-solid interaction (FSI) is frequently encountered in geotechnical engineering. Its complexity stems from the interaction between pore fluid and grains which consist of geomaterials like soil and rock. Liquefaction, soil erosion and sand production are typical geomechanical problems which involve strong pore fluid-particle coupling. However, microscopic investigations for such kind of problems in laboratory experiments are extremely hard due to the limitation of current techniques. As an alternative, the numerical method, like the discrete element method (DEM) [1] or bonded particle method (BPM) [2], has been proved to be useful for modeling geomaterials at the 
grain level. Since the 1980s, there have been several fluid approaches proposed for solving pore fluid flow and its coupling with solid particles in the framework of DEM.

Hakuno and Tarumi [3] first combined DEM and Darcy fluid flow (DFF) to analyze liquefaction of saturated sands under seismic excitation. In this method the excess pore water pressure was taken into account by tracing the change of individual pore volumes formed by neighboring particles, and the dissipation of excess pore water pressure was accomplished by two permeability coefficients for water moving through pores. However, only the tendency of gradually increased excess pore water pressure was captured and the fully liquefaction condition was not observed. In order to avoid the complicated individual pore volume calculation, an alternative method was proposed to consider the generation of excess pore water pressure at cell level rather than at pore level [4]. Applications of DEM-DFF in undrained behaviors of saturated sands and upward seepage were performed by Shafipour and Soroush [5] and Goodarzi et al. [6]. However, this continuum-discrete model is only applicable to the fluid-particle system with a small Reynolds number. The pore volume change is approximately calculated using mean relative displacements of particles in the four neighboring cells rather than direct treatments at the pore level. An advanced continuum-discrete model, which couples the computational fluid dynamics (CFD) and DEM, was proposed in chemical engineering by Tsuji et al. in 1992 [7]. In this technique, the fluid flow is characterized by Navier-Stokes equations. The hydrodynamic forces applied to solid particles can be computed through the configuration and velocities of solid particles. Then, this method is borrowed and applied to investigate soil liquefaction [8], particle movement in fluid [9,10] and sand production [11]. The computational cost of this coupled technique is less expensive due to the coarse fluid grid, where a set of semi-empirical equations are required to compute hydrodynamic forces applied on each particle.

To resolve some of the problems aforementioned, a fully discrete-discrete technique, where DEM and lattice Boltzmann method are combined, was proposed by Cook et al. [12]. The fluid phase is treated as an assembly of fluid particles whose movement is governed by the lattice Boltzmann equation. The fluid-particle interaction can be directly calculated through the momentum theorem. Detailed development of the coupling schemes used in DEMLBM was introduced in our previous work $[13,14]$. Applications of this hybrid technique in hydraulic fracture $[15,16]$, sand production [17], soil erosion [18,19], liquefaction [20] and other problems [21,22] can be found in the literature.

There are several coupling schemes proposed to solve fluid-particle interactions. Such as the modified half-way bounce back rule $[43,44]$, the interpolation-based approach [22,27,45], the immersed boundary method (IBM) [2325] and immersed moving boundary (IMB) [26,28] scheme. Particularly, IBM and IMB are most commonly adopted in LBM. The immersed boundary method was first proposed by Peskin in 1977 [29]. The basic idea of the IBM is to treat the particle boundary as a deformable body with high stiffness. Moving boundaries are represented by a set of boundary nodes. A small distortion of the particle boundary caused by the fluid-particle interactions will generate a force that tends to restore the particle to its original shape. The deformation is calculated by comparing the boundary point and the reference point that undergoes rigid body motions with particles. This penalty-based IBM was first introduced to lattice Boltzmann method through suitably adding a body force density term into flow 
governing equations by Feng and Michaelides in 2004 [23]. The no-slip condition at the fluid-particle interface is satisfied by calculating the velocity of particle boundary points through interpolating fluid velocities on neighboring nodes. Then, momentum exchange and direct forcing-based IBM [31,32] are proposed. It is reported that in the previous IB-LBM the non-slip boundary condition is not fully enforced due to explicit nature of forcing term formulation. Then, $\mathrm{Wu}$ and Shu [24] improved the IB-LBM using an implicit force density formulation where an unknown velocity correction is prescribed. The velocity correction was determined in such a way that the velocity at the boundary interpolated from the corrected velocity field satisfies the non-slip boundary condition. This implicit scheme requires complex matrix inversion and higher computational memory space. Recently, Dash et al., [25] proposed an implicit flexible forcing IBM by combining the implicit IB-LBM with a fixed multi-direct forcing IBM [33]. Instead of fixed iteration number in the fixed multi-direct forcing IBM, a flexible iteration for velocity correction is terminated when convergence limit is satisfied. This is the latest improvement of IB-LBM, especially in terms of computational efficiency.

The aims of this work are developing a DEMLBM coupled by the latest IBM [25], and investigating its applicability and accuracy for fluid-particle coupling. The remainder of this paper is organized as follows: Section 2 will briefly introduce the formulation and algorithm of IBM-LBM. It is followed by numerical tests and validations including comparison of interstitial fluid flow in porous media with semi-empirical Ergun equation and comparison of IBM with FEM and IMB in single particle sedimentation and migration tests. Because this paper is not about the application of DEMLBM coupled by IBM in geomechanics, complex simulations involving many particles are not given here but can be found in others' work [30,33]. Then discussions on the advantages and limitations of the latest IBM for DEMLBM will be given. Finally, conclusions will be drawn based on above investigations.

\section{Methodologies}

\subsection{Implicit flexible forcing IBM}

The lattice Boltzmann method is a modern numerical approach in computational fluid dynamics. In LBM the fluid domain is divided into regular lattices and the fluid phase is represented by a group of (imaginary) fluid particle packages resided at each lattice node. Each fluid particle package includes several fluid particles, such as 9 fluid particles in the commonly used D2Q9 model. The fluid flow can be achieved through resolving the particle collision and streaming processes, and the lattice Boltzmann equation is used to solve the streaming and collision processes of fluid particles. Primary variables of LBM are the so-called fluid density distribution functions, which are portions of the fluid density, associated with the fluid particles. Both mass and momentum of fluid particles are characterized by the fluid density distribution functions. The detail of LBM can be referred to [34, 35].

The lattice Boltzmann equation [40] considering body force is given by

$$
f_{i}\left(x+\mathbf{e}_{\mathbf{i}} \Delta t, t+\Delta t\right)-f_{i}(x, t)=\Omega_{i}+F_{i} \Delta t
$$


where $f_{i}$ is the fluid density distribution function in $i$ direction; $x$ and $\mathbf{e}_{\mathbf{i}}$ are the coordinates and velocity vectors (see Fig. 1) at the current lattice node; $t, \Omega_{\mathrm{i}}$ and $F_{i}$ are, respectively, the current time, collision operator and body force term. The 9 velocity vectors, $\mathbf{e}_{\mathbf{i}}$, in D2Q9 (see Fig. 1) are defined as

$$
\begin{gathered}
\mathbf{e}_{0}=(0,0) \\
\mathbf{e}_{\mathrm{i}}=C\left(\cos \frac{\pi(i-1)}{2}, \sin \frac{\pi(i-1)}{2}\right) \quad(\mathrm{i}=1, \ldots, 4) \\
\mathbf{e}_{\mathbf{i}}=\sqrt{2} C\left(\cos \frac{\pi(2 i-9)}{4}, \sin \frac{\pi(2 i-9)}{4}\right) \quad(\mathrm{i}=5, \ldots, 8)
\end{gathered}
$$

in which $C$ is the lattice speed and is related to the lattice spacing, $h$, and the time step, $\Delta t$

$$
C=h / \Delta t
$$

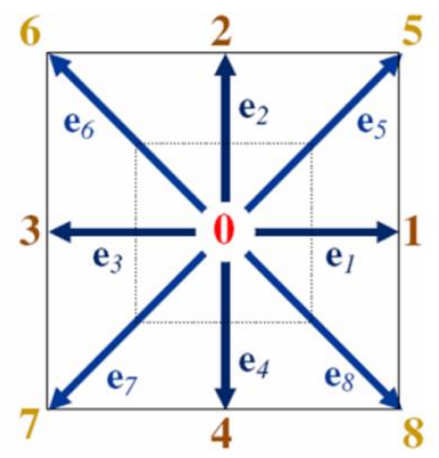

Fig. 1 D2Q9 model

There are mainly two collision operators and they are known as the Bhatnagar-Gross-Krook (BGK) [35] and multiple relaxation time (MRT) [41] collision operators. In this work, only the BGK model will be used. For the detailed introduction of MRT model, readers are supposed to refer to the relevant literatures [41,42]. In the single relaxation lattice BGK Model, $\Omega_{\mathrm{i}}$ is characterized by a relaxation time $\tau$ and the equilibrium distribution functions $f_{i}^{e q}(x, t)$

$$
\Omega_{i}=-\frac{\Delta t}{\tau}\left[f_{i}(x, t)-f_{i}^{e q}(x, t)\right]
$$

In this work, the D2Q9 model is adopted, and $f_{i}^{e q}(x, t)$ are defined as:

$$
f_{i}^{e q}=\omega_{i} \rho\left(1+\frac{3}{C^{2}} \mathbf{e}_{\mathbf{i}} \cdot \mathbf{u}+\frac{9}{2 C^{4}}\left(\mathbf{e}_{\mathbf{i}} \cdot \mathbf{u}\right)^{2}-\frac{3}{2 C^{2}} \mathbf{u} \cdot \mathbf{u}\right) \quad(i=0, \ldots, 8)
$$

where $\rho$ and $\mathbf{u}$ are the macroscopic fluid density and velocity, respectively. $\omega_{i}$ are the weighting factors:

$$
\omega_{0}=\frac{4}{9}, \quad \omega_{1,2,3,4}=\frac{1}{9}, \quad \omega_{5,6,7,8}=\frac{1}{36}
$$

The body force term is given by 


$$
\mathbf{F}_{\mathbf{i}}=\left(1-\frac{1}{2 \tau}\right) w_{i}\left(\frac{\mathbf{e}_{\mathbf{i}}-\mathbf{u}}{C_{S}^{2}}+\frac{\mathbf{e}_{\mathbf{i}} \cdot \mathbf{u}}{C_{S}^{4}}\right) \cdot \mathbf{f}
$$

where $C_{S}$ is the speed of sound in fluid, defined as $C_{S}=C / \sqrt{3}$. $\mathbf{f}$ is the force density at the lattice node and its computation will be given later.

The corresponding fluid velocity is modified by force density as

$$
\rho \mathbf{u}=\sum_{i=1}^{8} f_{i} \mathbf{e}_{\mathbf{i}}+\frac{1}{2} \mathbf{f} \Delta t
$$

$\rho$ is macroscopic fluid density and given by

$$
\rho=\sum_{i=0}^{8} f_{i}
$$

The fluid pressure is given by

$$
\Delta P=C_{S}^{2} \Delta \rho
$$

In the application of LBM, it is more convenient to choose $\tau$ and $h$ as two independent parameters, then the time step, $\Delta t$, can be computed by

$$
\Delta t=\left(\tau-\frac{1}{2}\right) \frac{h^{2}}{3 v}
$$

where $v$ is fluid viscosity.

It is obvious from Equation (11) that $\tau$ should be greater than 0.5, and Equation (10) reveals that the incompressibility of the fluid is not exactly enforced. The LBM can be viewed as a 'penalty-based' method that allows a limited degree of compressibility to occur where the speed of sound in fluid acts as a penalty value.

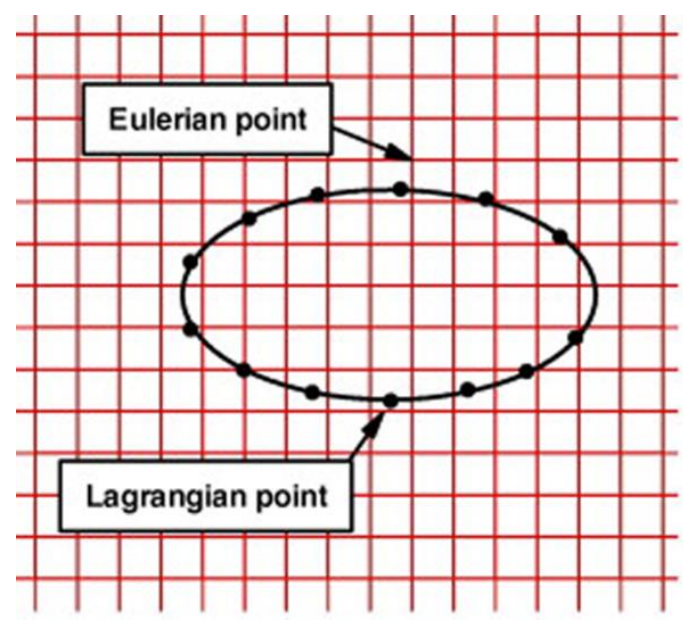

Fig. 2 Immersed boundary scheme (after [24]) 
In IBM (see Fig. 2), the solid boundary is discretized into several Lagrangian boundary points. In each LBM time step, the following IBM steps will be performed.

1) Calculate the velocity of boundary points of each particle through rigid body motion;

2) Calculate the fluid velocity at the same boundary nodes through the interpolation of neighbouring fluid nodes using Dirac delta distribution functions;

3) Compute the difference (so-called velocity correction, $\delta \mathbf{U}_{\mathbf{b}}$ ) between the interpolated boundary velocity and the velocity of boundary points;

4) The velocity correction of each boundary point is then distributed to its surrounding fluid nodes using the following equation

$$
\delta \mathbf{u}\left(x_{i j}, t\right)=\sum_{k} \delta \mathbf{U}_{\mathbf{b}}\left(x_{b}^{k}, t\right) \cdot D\left(x_{i j}-x_{b}^{k}\right) \cdot \Delta s_{k}
$$

$x_{i j}$ and $x_{b}^{k}$ are the coordinates of fluid nodes and boundary points, $k$ is the serial number of boundary points related to the fluid nodes and $\Delta s$ is the arc length between two consecutive boundary points. $D(\cdots)$ is a Dirac delta function.

5) The velocity and density force at the lattice node will be calculated by

$$
\begin{gathered}
\mathbf{u}\left(x_{i j}, t\right)=\mathbf{u}\left(x_{i j}, t\right)+\delta \mathbf{u}\left(x_{i j}, t\right) \\
\mathbf{f}=\mathbf{f}+\frac{2 \rho \delta \mathbf{u}\left(x_{i j}, t\right)}{\Delta t}
\end{gathered}
$$

6) Meanwhile, the hydrodynamic force applied to the boundary point can be computed

$$
\mathbf{F}_{\mathbf{b}}\left(x_{b}, t\right)=\mathbf{F}_{\mathbf{b}}\left(x_{b}, t\right)+\frac{2 \rho \sum_{k} \delta \mathbf{U}_{\mathbf{b}}\left(x_{b}^{k}, t\right)}{\Delta t}
$$

7) Repeat steps 2) to 6) for each boundary node until the velocity correction at the boundary point is small enough $\left(<10^{-5}\right)$.

8) Then, update fluid density distribution functions using Eq. (1) for next time step and fluid velocity using Eq. (8).

It is noticed that Eq. 13 is used to update the lattice velocity around the solid boundary point, and the updated velocity will be used to interpolate the fluid velocity at the solid point in step 2). Generally, this equation is only temporarily used to obtain converged results. For the fluid nodes around a solid boundary point, Eqs 8 and 13 should be equivalent since the force density used in Eq. 8 is continuously updated. Particularly, Eq. 8 can be used to update velocities of all fluid nodes regardless of it is surrounding solid boundary points or not. 
In DEM the movement of each particle is updated by the Newton's second law:

$$
\begin{aligned}
m \mathbf{a}+c \mathbf{v} & =\mathbf{F}_{\mathbf{c}}+\mathbf{F}_{\mathbf{f}}+m \mathbf{g} \\
\ddot{\boldsymbol{\theta}} & =\mathbf{T}_{\mathbf{c}}+\mathbf{T}_{\mathbf{f}}
\end{aligned}
$$

where $m$ and $I$ are respectively the mass and the moment of inertia of particles, $c$ is a damping coefficient, $\mathbf{a}$ and $\ddot{\boldsymbol{\theta}}$ are acceleration and angular acceleration, $\mathbf{F}_{\mathbf{c}}$ and $\mathbf{T}_{\mathbf{c}}$ are, respectively, contact forces and corresponding torques, $\mathbf{F}_{\mathrm{f}}$ and $\mathbf{T}_{\mathrm{f}}$ are hydrodynamic forces and corresponding torques. Lubrication force is not considered in this work.

The contact force is obtained by a linear spring model. Details of the contact model used in this work and calculation of time step can be found in our previous work [13,34]. Time integration of DEM is achieved by the central difference method.

When coupling DEM with LBM, a sub-cycling algorithm is employed. In general the time step of DEM is smaller than the one of LBM. Therefore in one LBM time step, $N_{s}$ sub-steps will be performed for DEM simulations.

$$
N_{s}=\frac{\Delta t}{\Delta t_{D E M}}
$$

where $\Delta t_{D E M}$ is the time step of DEM.

The coupling process between DEM and IBM-LBM can be found in Fig. 3. It should be highlighted that DEM iteration is only performed when the velocity correction at the boundary point converges during the coupling process.

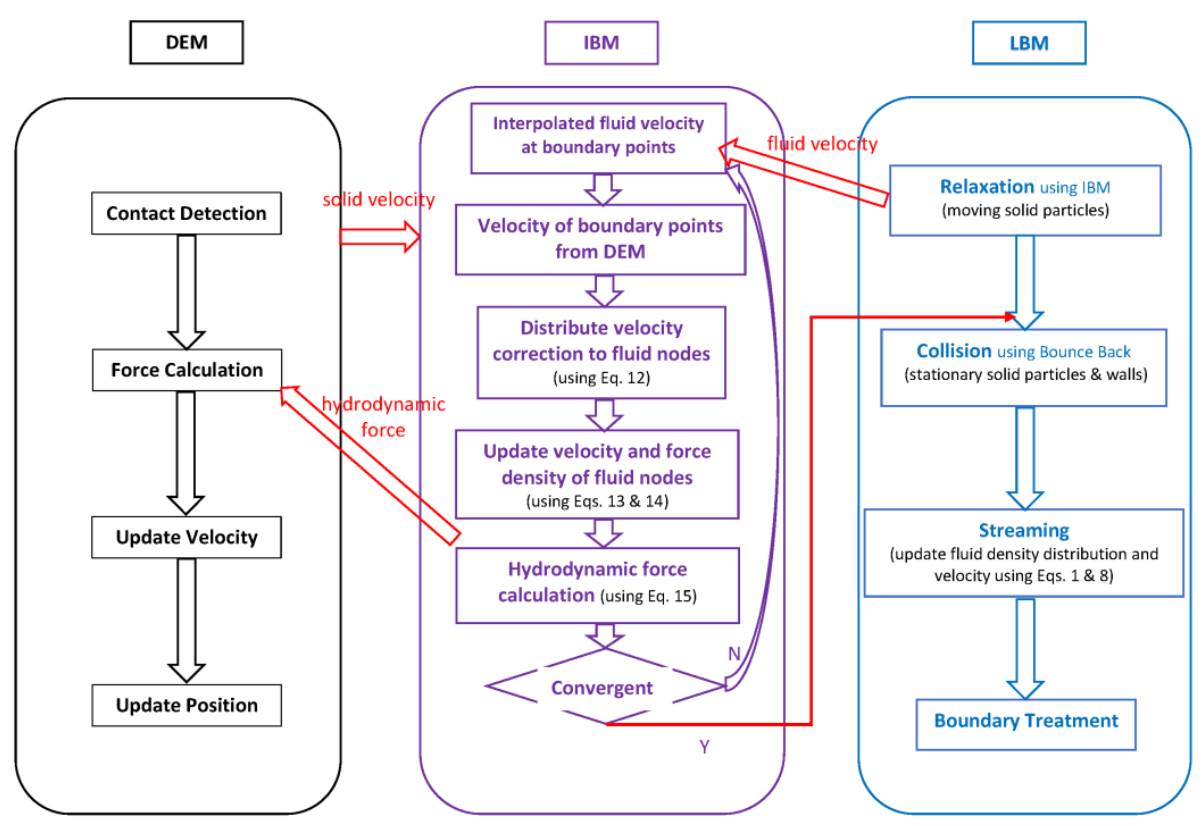

Fig. 3 Coupling of DEM-LBM by IBM 


\subsection{Immersed moving boundary scheme}

For the sake of completeness, a brief introduction of the immersed moving boundary scheme [26] is given below. In this method, a particle is represented by the solid (lattice) nodes which are located within the particle. A solid node is called interior if its linked nodes are all solid nodes, while if a solid has at least one link to a fluid node, it is called a solid boundary node. A fluid node having at least one link to a solid node is defined as the fluid boundary node. Thus, there are four types of node in the IMB scheme: interior solid node, solid boundary node, fluid boundary node and normal fluid node, which are respectively marked in red, yellow, green and blue in an illustrative diagram of IMB in Fig. 4. In order to retain the advantages of LBM, namely the locality of the collision operator and the simple linear streaming operator, an additional collision term, $\Omega_{i}^{S}$, for the boundary nodes covered partially or fully by the solid is introduced to the standard collision operator of LBM. The modified collision operator for resolving the fluid-solid interaction on IMB is given by

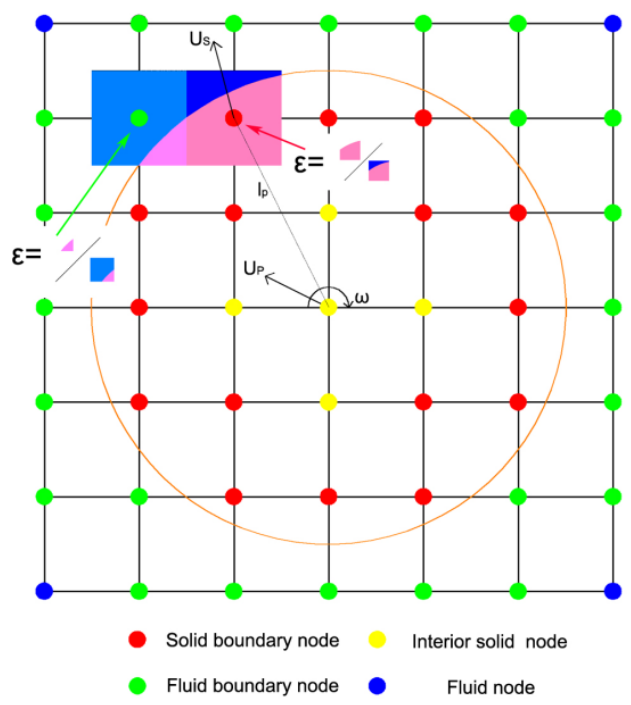

Figure 4 IMB scheme

$$
\Omega=-\frac{\Delta t}{\tau}(1-B)\left[f_{i}(x, t)-f_{i}^{e q}(x, t)\right]+(1-B) \Delta t \mathbf{F}_{\mathbf{i}}+B \Omega_{i}^{S}
$$

where $\mathbf{F}_{\mathbf{i}}$ is a body force term,$B$ is a weighting function that depends on the local solid ratio $\varepsilon$, defined as the fraction of the node area (see Fig. 4). It should be highlighted that the weighting function could smoothly represent the solid boundary during movement.

$$
B=\frac{\varepsilon(\tau-0.5)}{(1-\varepsilon)+(\tau-0.5)}
$$

When $\varepsilon=0, \mathrm{~B}=0 ; \varepsilon=1, \mathrm{~B}=1$.

The additional collision term is based on the bounce-rule for the non-equilibrium part

$$
\Omega_{i}^{S}=f_{-i}(x, t)-f_{i}(x, t)+f_{i}^{e q}\left(\rho, \mathbf{U}_{\mathbf{S}}\right)-f_{-i}^{e q}(\rho, \mathbf{u})
$$


where $\mathbf{U}_{\mathbf{S}}$ is the velocity of the solid node (see Fig. 1) and $\mathbf{u}$ is the velocity of the fluid at the node.

The resultant hydrodynamic force and torque exerted on the solid particle can be calculated by

$$
\begin{gathered}
\mathbf{F}_{f}=C h\left[\sum_{n}\left(B_{n} \sum_{i} \Omega_{i}^{s} \mathbf{e}_{i}\right)\right] \\
\mathbf{T}_{f}=\operatorname{Ch}\left\{\sum_{n}\left[\left(x-x_{P}\right) \times\left(B_{n} \sum_{i} \Omega_{i}^{s} \mathbf{e}_{i}\right)\right]\right\}
\end{gathered}
$$

\section{Numerical examples and validations}

\subsection{Interstitial fluid flow in porous media}

The flow of interstitial fluid in porous media has been extensively investigated in different disciplines since the 1950s [36,37]. In this section, the interstitial fluid flow in regularly and densely packed particles will be carried out first, and the well-known semi-empirical Ergun equation will be utilized to validate numerical results. The numerical set-up is shown in Fig. 5. The fluid domain is divided into $200 \times 400$ square lattices with spacing $\mathrm{h}=0.1 \mathrm{~mm}$. The kinematic viscosity and density of the fluid are $10^{-6} \mathrm{~m}^{2} / \mathrm{s}$ and $1000 \mathrm{~kg} / \mathrm{m}^{3}$, respectively. The relaxation time $(\tau)$ in LBM is select as 0.5001 , and time step is $3.33 \times 10^{-7} \mathrm{~s}$. The regular packing includes 200 disks of radius $\mathrm{R}=1 \mathrm{~mm}$. The density of the solid particle is $3000 \mathrm{~kg} / \mathrm{m}^{3}$. A periodic boundary condition [46] is applied to the left inlet and right outlet. Through applying different pressure drops, the relation between pressure drop and superficial velocity can be obtained. Because the flow path in 2D simulations is blocked by solid particles, an effective radius of solid particles is required to simulate real fluid flow. Effective particle radius used in this work is $0.85 \mathrm{R}$. Then true porosity is 0.43255 . The equilibrium fluid flow of IBM with 20 boundary points and IMB simulations with $0.85 \mathrm{R}$ is given in Fig. 6 which is zoomed in from the local domain enclosed by yellow line in Fig. 5. The analytical superficial velocity $v_{S}$ under different pressure drops $\Delta p$ across the packing can be calculated by [36]

$$
\Delta p=150 \mu L v_{S} \frac{(1-n)^{2}}{D_{p}^{2} n^{3}}+1.75 L \rho v_{s}^{2} \frac{(1-n)}{D_{p} n^{3}}
$$

where $\mu$ is the dynamic viscosity of fluid, $L$ is the packing length, $D_{p}$ is the diameter of particles, $n$ is the porosity of the packing.

Then comparison between gradient of pressure drop and superficial velocity obtained by IBM with 20 boundary points, IMB and Ergun equation are given in Fig. 7. The result of IBM, IMB and analytical solution are respectively marked by black triangle, blue cross and red line. It is found that when fluid superficial velocity is lower than 0.01 $\mathrm{m} / \mathrm{s}$, numerical results obtained by IBM and IMB match Ergun equation very well. With the increase of fluid velocity, deviation grows up.

The LBM can be viewed as a 'penalty-based' method that allows a limited degree of compressibility, and the compressibility error is measured by Mach number $(M a)$ 


$$
M a=\frac{u_{\max }}{C}
$$

where $u_{\max }$ is the maximum fluid velocity in the fluid simulations. It can be found that the compressibility error is proportional to the fluid velocity. This could be one possible reason why difference between simulations and Ergun equation increases with fluid superficial velocity.

The modified Reynolds number $\left(G r_{p}\right)$ is an important dimensionless quantity in fluid mechanics to measure flow patterns in different fluid flow situations. It is positively proportional to fluid velocity and can be calculated by

$$
G r_{p}=\frac{\rho v_{S} D_{p}}{(1-n) \mu}
$$

Normally, the friction factor $\left(f_{p}\right)$ as a function of the modified Reynolds number $\left(G r_{p}\right)$ is a standard index in a packed column. It can be determined by

$$
f_{p}=\frac{150}{G r_{p}}+1.75
$$

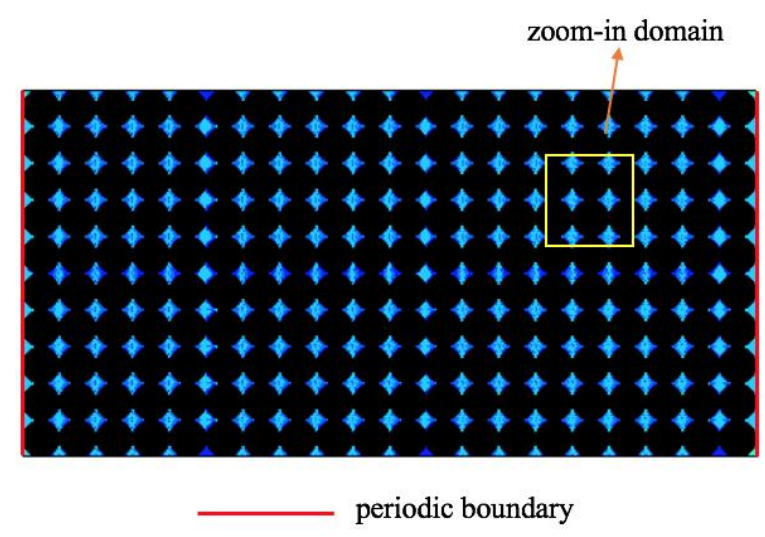

Fig. 5 Set-up of interstitial fluid flow

Fig. 8 shows the friction factor - modified Reynolds number curves. We can see that the numerical results of IBM and IMB generally match the analytical solution. There is certain difference between them when Reynolds number increases. However, it is less obvious than that between pressure drop and modified Reynolds number. 

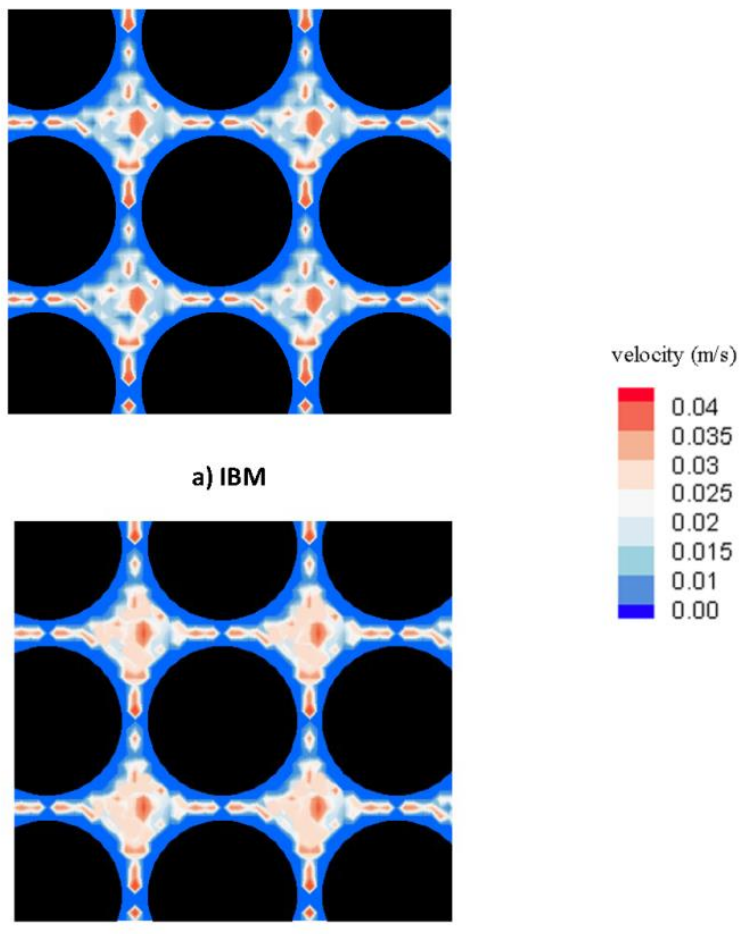

b) IMB

Fig. 6 Fluid velocity contour at equilibrium with $0.85 \mathrm{R}$

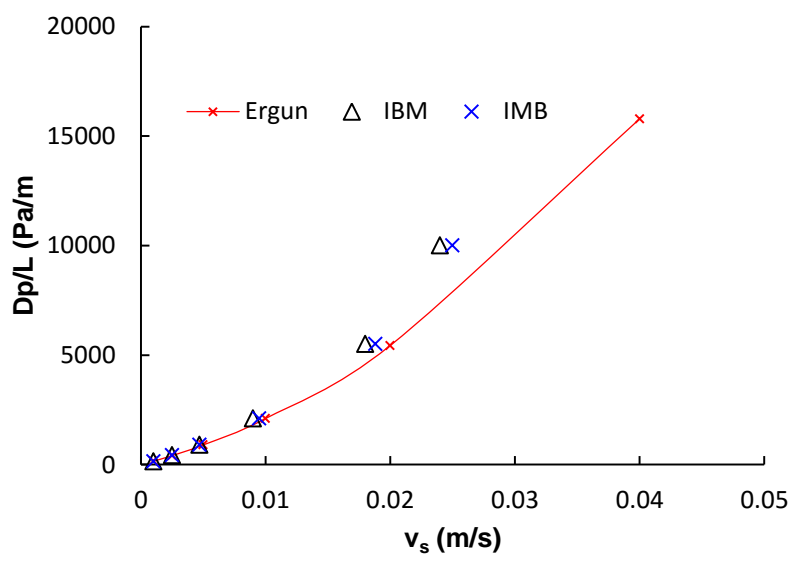

Fig. 7 Gradient of pressure drop versus superficial velocity 


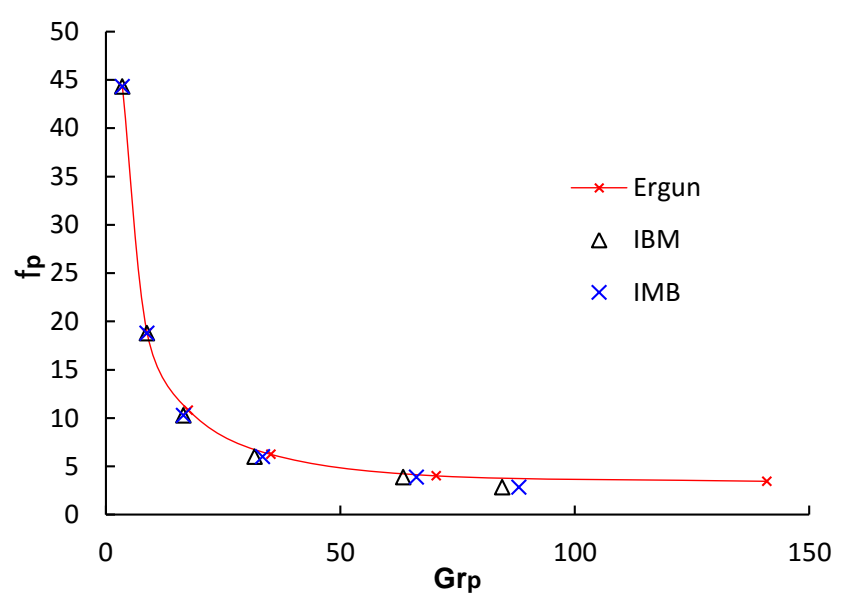

Fig. 8 Relation between friction factor and modified Reynolds number

\subsection{Single particle sedimentation in fluid}

The second benchmark is single particle sedimentation in a box filled by viscous fluid [47]. For comparison, this model will be carried out separately by IBM and IMB. The dimension of the box is $2 \mathrm{~cm}$ (in the X-direction) by 6 $\mathrm{cm}$ (in the Y-direction). The fluid domain is divided into $200 \times 600$ square lattices with spacing $\mathrm{h}=0.1 \mathrm{~mm}$. The viscosity and density of the fluid are $0.1 \mathrm{~Pa} \cdot \mathrm{s}$ and $1 \mathrm{~g} / \mathrm{cm}^{3}$, respectively. The density of the solid particle is $1.25 \mathrm{~g} / \mathrm{cm}^{3}$, and its radius is $1.25 \mathrm{~mm}$. The relaxation time $(\tau)$ in LBM is select as 0.8 , and the time step is $0.0001 \mathrm{~s}$. Four boundaries of the model are stationary walls and thus the no-slip boundary condition is imposed for the fluid. Initially, one stationary solid particle is generated at the position $(1 \mathrm{~cm}, 4 \mathrm{~cm})$. Due to gravity, the solid particle will move downward gradually. First the IBM is employed for solving fluid-particle interaction, and surface of the solid particle is divided into 5, 10, 20 and 25 boundary points in different simulations. For comparison, the extensively used IMB is utilized to run the same test case as well. In these tests, the size ratio of particle diameter to lattice spacing is 25 . The effect of lattice spacing on numerical accuracy of IMB has been investigated by some researchers $[38,39]$ and a size ratio 20 is reported as the minimum value to produce relatively accurate simulations.

Particle movement and fluid velocity contours at different instants, 0.1 s, 0.4 s, 0.6 s, 0.8 s, 0.9 s, are given in Fig. 9. Fig. 10 compares the vertical movement of the solid particle in IBM simulations with FEM and IMB simulations. It is found that the solid particle settles in an accelerated manor in the initial stage. After $0.2 \mathrm{~s}$, the vertical velocity reaches an equilibrium state (see Fig. 11). The results of IBM with 25 boundary points and results of IMB match the FEM simulations performed by Wan and Turek [47]. From Fig. 10, we can see the movement of the solid particle obtained by IBM with less boundary points (e.g. 5, 10, and 20) are delayed. With the increase of boundary points, the simulation is approaching to the results of FEM and IMB. It can be confirmed by the variation of velocity of solid particle with the number of boundary points (NBP) 5, 10, 20 and 25 shown in Fig. 11. With the increase of number of boundary points, the magnitude of equilibrium velocity increases. The difference observed is caused by the hydrodynamics forces which are shown in Fig. 12. It is noticed that with the decrease of boundary points the fluctuation of hydrodynamic forces increases. It seems the IBM with less NBP yields larger hydrodynamics force 
which is opposite to the gravity, therefore the downward movement is delayed. Large repulsion in IBM with $\mathrm{NBP}=25$ and IMB can be observed around $0.8 \mathrm{~s}$ when the solid particle is close or in collision with the bottom wall. Generally, apparent fluctuation of the hydrodynamic force computed by IBM is found compared to the one computed by IMB. However, a good agreement between IBM with 25 NBP and IMB can be found.
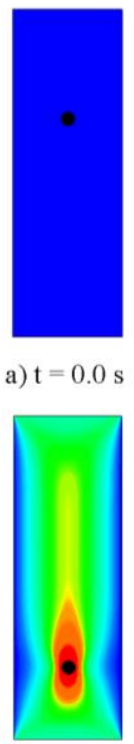

d) $\mathrm{t}=0.6 \mathrm{~s}$

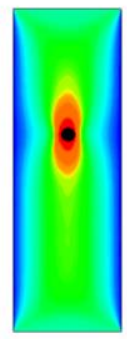

b) $\mathrm{t}=0.1 \mathrm{~s}$

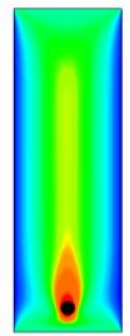

e) $\mathrm{t}=0.8 \mathrm{~s}$

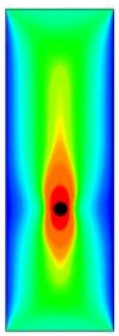

c) $\mathrm{t}=0.4 \mathrm{~s}$

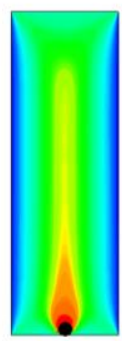

f) $\mathrm{t}=0.9 \mathrm{~s}$

Fig. 9 Particle movement and fluid velocity contours at different instants $(\mathrm{NBP}=25)$

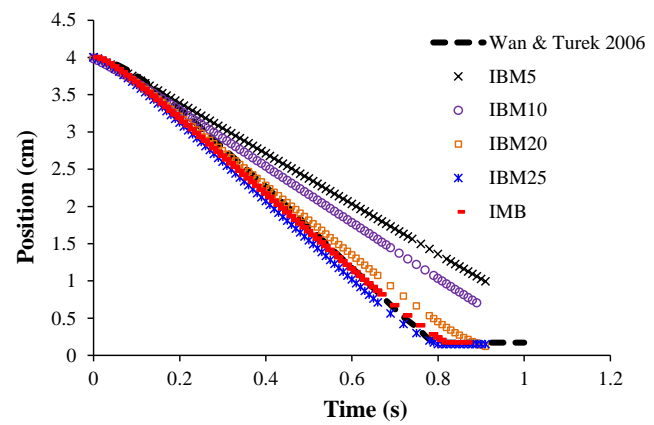

Fig. 10 Comparison of vertical position of solid particle in IBM, FEM and IMB simulations

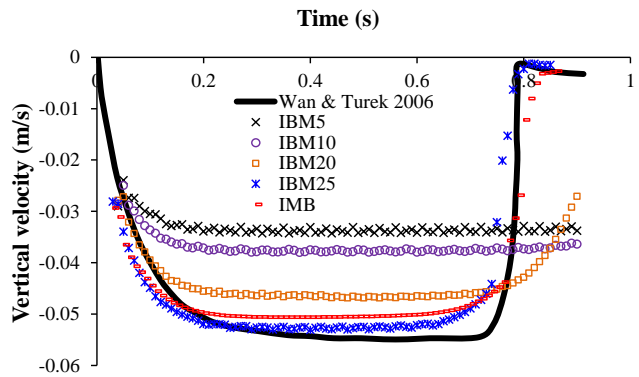

Fig. 11 Variation of vertical velocity of solid particle over time with different boundary points 


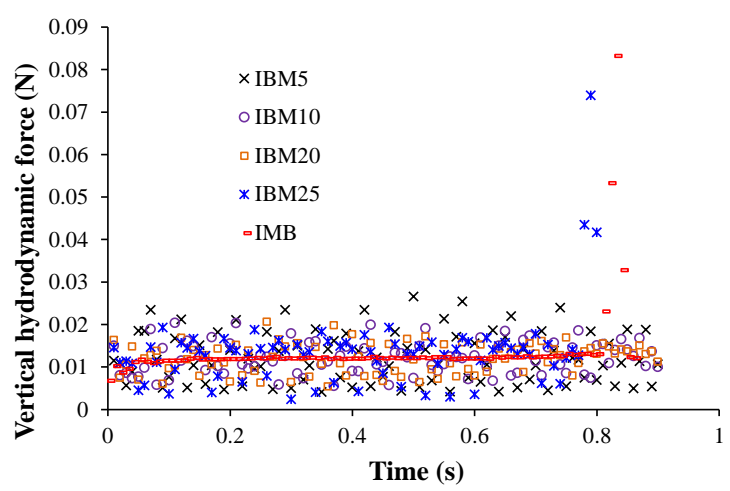

Fig. 12 Comparison of hydrodynamic forces applied on solid particle by IBM and IMB

\subsection{Migration of particle in Poiseuille flow}

To further examine the accuracy of IBM, the migration of one particle along a horizontal pipe in the Poiseuille flow condition is performed. To accelerate the computing speed, the periodic boundary is implemented in the streamwise direction by treating nodes on the inflow and outflow faces as nearest neighbors. In our simulation the inlet and outlet are on the left and right, respectively. Pressure drop between inlet and outlet is $30 \mathrm{~Pa}$. Stationary walls are set at both top and bottom boundaries. The dimensions of fluid domain are $8 \mathrm{~cm}$ in X-direction and $1 \mathrm{~cm}$ in Y-direction. Fluid domain is divided into $800 \times 100$ square lattices with spacing $\mathrm{h}=0.1 \mathrm{~mm}$. The kinematic viscosity $(\eta)$ and density of the fluid are $10^{-6} \mathrm{~m}^{2} / \mathrm{s}$ and $1000 \mathrm{~kg} / \mathrm{m}^{3}$, respectively. The relaxation time $(\tau)$ in LBM is selected as 0.50001 , and the time step is $3.33 \times 10^{-8} \mathrm{~s}$. The sub-iteration number $\left(N_{s}\right)$ for DEM is 1 . At the beginning the particle whose radius is $1.5 \mathrm{~mm}$ is placed at $(2 \mathrm{~cm}, 0.5 \mathrm{~cm})$. Simulations with different NBP are carried out. The size ratio of diameter $D_{p}$ to lattice spacing is 30 . Fig. 13 shows velocity contours of fluid at different stages. The maximum fluid velocity is $5.1 \mathrm{~m} / \mathrm{s}$ and the lattice speed is $300 \mathrm{~m} / \mathrm{s}$. The computed Mach number (see Eq. 25) is far smaller than 1.0. Hence the simulation is supposed to be reliable. The numerical results obtained by IBM and IMB schemes are compared in Figs. 14-16. Fig. 14 compares particle movement in horizontal direction. A good agreement of particle movement simulated by both methods can be observed only in the first half stage. With the increase of velocity of the solid particle, derivation between them keeps increasing with time. It is interesting that with the increase of boundary points derivation of horizontal position grows up. From Fig. 15 which gives the comparison of horizontal velocity of solid particle, we can find that velocity departs from an earlier (a quarter of) stage than position, and the velocity threshold is below $0.5 \mathrm{~m} / \mathrm{s}$. The difference of position and velocity is attributed to the computed hydrodynamic forces. Comparison of hydrodynamic forces applied to solid particle can be seen in Fig. 16. Similar to the particle sedimentation test, with the decrease of NBP the hydrodynamic force increases. Because the hydrodynamic force is the driving force of particle movement, the solid particle departs from IMB simulations when NBP increases. While in the sedimentation simulations, gravity is the driving force and hydrodynamic forces are 
opposite to particle movement. Hence the solid particle departs from IMB simulations when NBP decreases. It is seen within $\mathrm{t}=0.005 \mathrm{~s}$, the hydrodynamic force is consistent. The Reynolds number $(\mathrm{Re})$, corresponding to $\mathrm{t}=$ $0.005 \mathrm{~s}$, can be calculated as 1200 by Eq. 28 .

$$
\operatorname{Re}=\frac{u D_{p}}{\eta}
$$

Subsequently, oscillation of the hydrodynamic force computed by IBM initiates and the magnitude increases quickly. Besides, with the decrease of NBP the fluctuation magnitude increases. After the half stage of simulation, negative hydrodynamic forces appear. While, the hydrodynamic force computed by IMB grows in a relatively stable state, and no negative values and unreasonable fluctuation observed in IBM can be found. Obviously, negative hydrodynamic forces are not expected, because they drive the solid particle move toward the right outlet.
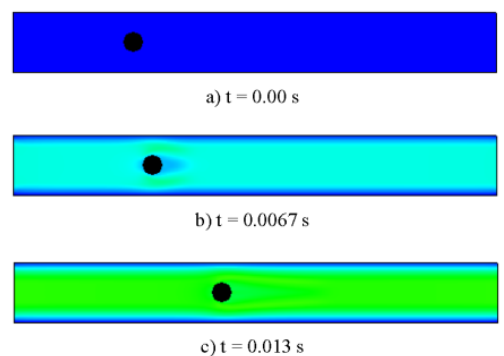

c) $\mathrm{t}=0.013 \mathrm{~s}$

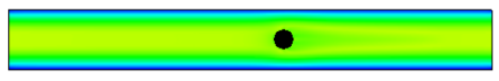

d) $\mathrm{t}=0.017 \mathrm{~s}$
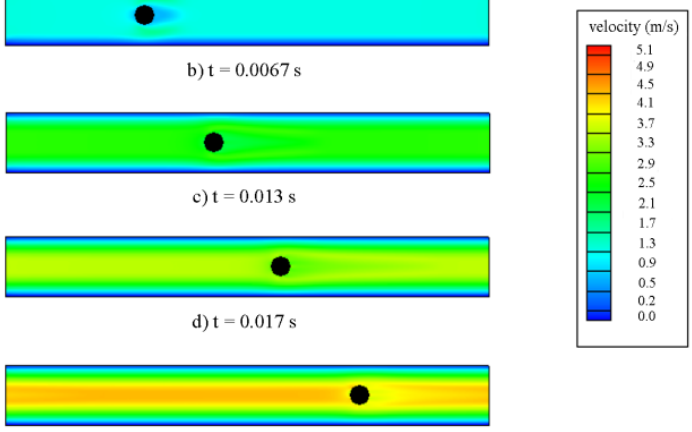

e) $\mathrm{t}=0.020 \mathrm{~s}$

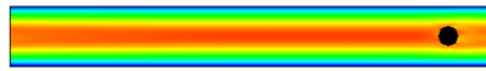

f) $t=0.023 \mathrm{~s}$

Fig. 13 Fluid velocity contour and particle position at different stages $(\mathrm{NBP}=20)$

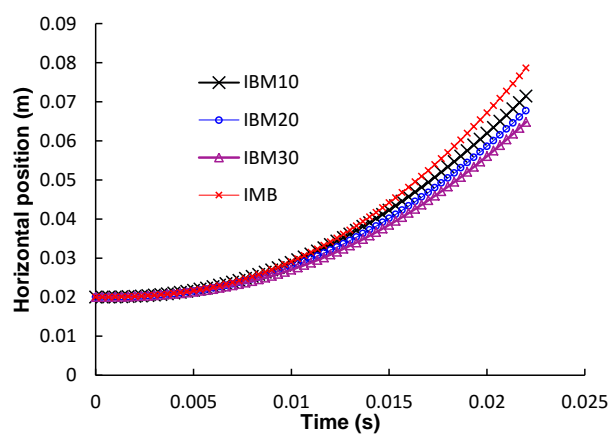

Fig. 14 Comparison of particle movement in $\mathrm{X}$ direction 


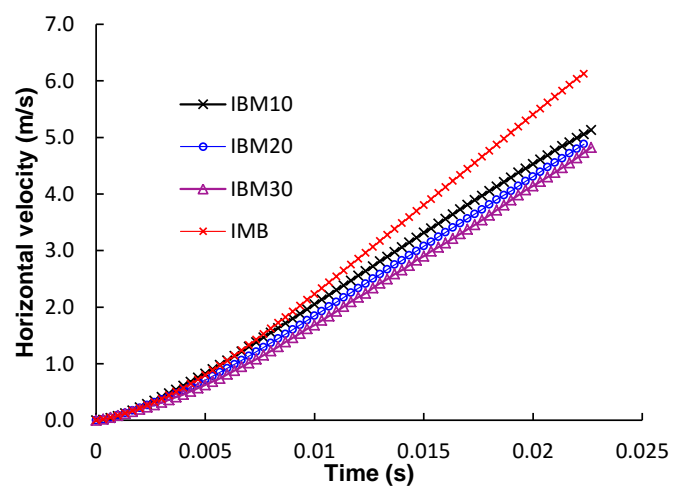

Fig. 15 Comparison of horizontal velocity of solid particle

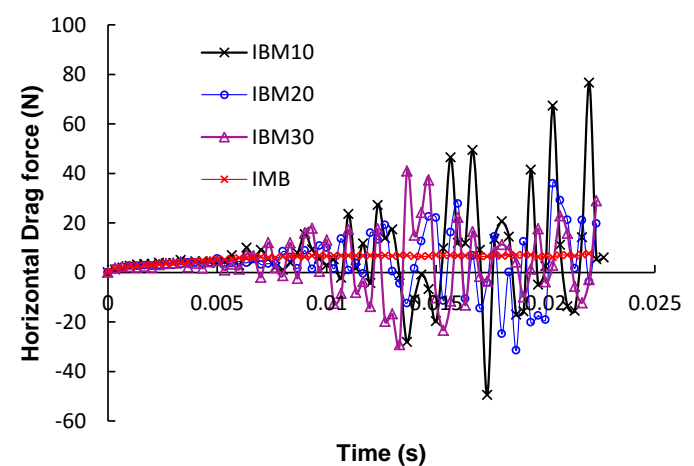

Fig. 16 Comparison of drag forces applied to solid particle

\section{Discussions}

From aforementioned simulations, the accuracy of IBM and its applicability in DEMLBM have been demonstrated. The simulation converges with the increase of NBP, and its accuracy can be only guaranteed when sufficient NBP are used. From our investigation, if more NBP, like 30 in the single particle sedimentation test, are adopted convergence criteria in step 7) will never be satisfied. Then, a problem how to select the NBP in different simulations comes up. Theoretically, each fluid grid should only hold one boundary point. From preliminary study, it is found the NBP can be selected as the size ratio of particle diameter to lattice spacing. Hence when NBP equals 25 an accurate simulation of particle sedimentation can be obtained. In addition, it should be highlighted that the IBM is not suitable to simulate problems involving large Reynolds number or particle velocity. This is confirmed by simulations of interstitial fluid flow in porous media, where only if Reynolds number is smaller than 70 numerical results match the semi-empirical Ergun equation, and migration of particle in Poiseuille flow, where only if particle velocity is less than $1 \mathrm{~m} / \mathrm{s}$ accurate modeling can be obtained. Even in single particle sedimentation simulations, where velocity of the solid particle is relatively small, oscillation of hydrodynamic forces can be found. One 
possible reason is the cell-crossing noise when a boundary point moves across lattice boundary. The hydrodynamic force applied on a boundary point is calculated through the velocity difference of nodes around the boundary point. When the boundary point moves from one grid to another, there will be a jump between the hydrodynamic forces calculated in the consecutive two time steps because the surrounding nodes used to calculate hydrodynamic forces are different. While in IMB, the boundary of solid can be accurate represented by the weighting function $B$ in Eq. 19, especially, when the solid particle moves. Fortunately, in lots of fluid-solid coupling problems in geomechanics, except sand production, debris flow and piping, the fluid velocity or particle movement is not very large.

Compared to IMB, the implementation of IBM in DEMLBM is not as difficult as IMB. The latter needs a good searching algorithm to efficiently identify different node types for each solid particle at each timestep [14]. Besides, the calculation of the solid ratio of area occupied by solid particles to fluid area at each node is another challenge in the implementation of IMB. While in IBM the hydrodynamic force can be directly calculated through implicit interpolation of fluid velocity. However, it seems the implicit interpolation of fluid velocity is time-consuming, especially in high velocity fluid flow. The following paragraph will give the computing resource used in this work and computing costs of simulations with IBM and IMB will be compared.

The information of the computer used is as follows: 1) Processor: Intel Core i5-6200 CPU@ 2.30GHz; 2) Memory: $8.00 \mathrm{~GB}$.

The computational costs of IBM and IMB in different tests are given in Table 1. It should be mentioned that in the above simulations by IMB the searching algorithm and the method for computing nodal solid ratio are the same as those used in our previous work [21]. A more efficient algorithm can be found in our latest work [14].

Table 1 Computational cost of IBM and IMB

\begin{tabular}{|c|c|c|}
\hline & Particle sedimentation & Migration in Poiseuille flow \\
\hline IBM (NBP=10) & $44 \mathrm{~m} \mathrm{05s}$ & $9 \mathrm{~h} 24 \mathrm{~m} \mathrm{22s}$ \\
\hline IBM (NBP=20) & $45 \mathrm{~m} 12 \mathrm{~s}$ & $11 \mathrm{~h} \mathrm{59m} \mathrm{38s}$ \\
\hline IBM $(\mathrm{NBP}=25)$ & $45 \mathrm{~m} 18 \mathrm{~s}$ & - \\
\hline IBM $(\mathrm{NBP}=30)$ & - & $12 \mathrm{~h} 43 \mathrm{~m} 51 \mathrm{~s}$ \\
\hline IMB & $42 \mathrm{~m} \mathrm{29s}$ & $2 \mathrm{~h} 43 \mathrm{~m} 31 \mathrm{~s}$ \\
\hline
\end{tabular}

Computing costs of particle sedimentation simulations using IBM and IMB are very similar, though the computing cost increases with NBP. The reason is that the fluid velocity is relatively small and the iteration number of velocity correction at each boundary point is less in sedimentation cases. While the computing time increases significantly with NBP in the simulations of particle migration in Poiseuille flow. It is found that even using our old IMB version, its computational efficiency is better than IBM. This difference is remarkable in high velocity fluid flow, in which the implicit IBM algorithm requires more iteration numbers in the convergence process of velocity correction. 


\section{Conclusions}

In this work, the latest implicit IBM was employed to couple DEM and LBM. Its accuracy, applicability and efficiency are examined through two benchmark tests and simulations of migration of particle in Poiseuille flow. Besides of the validation using semi-implicit Ergun equation, comparisons with the extensively used FEM and IMBLBM are also made. Then, the following conclusions can be drawn.

1) Accurate simulations of IBM-LBM can be achieved under certain limitations, like $\operatorname{Re}<1200$ and proper NBP, which is demonstrated through comparing results with Ergun equation, FEM and IMB simulations.

2) The effect of number of boundary points in IBM is remarkable. Its accuracy can only be guaranteed when sufficient NBP are used. It is found NBP can be selected as the size ratio of particle diameter to lattice spacing.

3) Compared to IMB, numerical implementation of IBM in DEMLBM is easier, but its computational efficiency is inferior. This problem is more obvious in fluid flow simulations with high velocity.

4) It is found that IBM is not suitable to simulate geomechanical problems involving large fluid or particle velocity, where unexpected oscillation of hydrodynamic forces is observed. Even in the simulation of single particle sedimentation where the particle velocity is not large, fluctuation of hydrodynamic forces can also be found.

The above findings are only valid for two-dimensional simulations. Further validations in three-dimensional problems will be performed in the near future.

\section{References}

[1] Cundall, P.A. and Strack, O.D., 1979. A discrete numerical model for granular assemblies. geotechnique, 29(1), pp.47-65.

[2] Potyondy, D.O. and Cundall, P.A., 2004. A bonded-particle model for rock. International journal of rock mechanics and mining sciences, 41(8), pp.1329-1364.

[3] Hakuno, M. and Tarumi, Y., 1988. A granular assembly simulation for the seismic liquefaction of sand. Doboku Gakkai Ronbunshu, 398, pp.129-138.

[4] Iwashita, K. and Oda, M., 1999. Mechanics of granular materials: an introduction. CRC press.

[5] Shafipour, R. \& Soroush, A. 2008. Fluid coupled-DEM modelling of undrained behavior of granular media. Computers and Geotechnics, 35, 673-685.

[6] Goodarzi, M., Kwok, C. Y. \& Tham, L. G. 2015. A continuum-discrete model using Darcy's law: formulation and verification. International Journal for Numerical and Analytical Methods in Geomechanics, 39, 327-342

[7] Tsuji, Y., Tanaka, T. \& Ishida, T. 1992. Lagrangian numerical simulation of plug flow of cohesionless particles in a horizontal pipe. Powder Technology, 71, 239-250. 
[8] Zeghal, M. and El Shamy, U., 2004. A continuum- discrete hydromechanical analysis of granular deposit liquefaction. International Journal for Numerical and Analytical Methods in Geomechanics, 28(14), pp.1361-1383.

[9] Jing, L., Kwok, C.Y., Leung, Y.F. and Sobral, Y.D., 2016. Extended CFD-DEM for free- surface flow with multi- size granules. International journal for numerical and analytical methods in geomechanics, 40(1), pp.62-79.

[10] Li, X. and Zhao, J., 2018. A unified CFD- DEM approach for modeling of debris flow impacts on flexible barriers. International Journal for Numerical and Analytical Methods in Geomechanics, 42(14), pp.1643-1670.

[11] Climent, N., Arroyo, M., O’Sullivan, C. and Gens, A., 2014. Sand production simulation coupling DEM with CFD. European Journal of Environmental and Civil Engineering, 18(9), pp.983-1008.

[12] Cook, B.K., Noble, D.R. and Williams, J.R., 2004. A direct simulation method for particle-fluid systems. Engineering Computations, 21, pp.151-168.

[13] Feng, Y.T., Han, K. and Owen, D.R.J., 2007. Coupled lattice Boltzmann method and discrete element modelling of particle transport in turbulent fluid flows: Computational issues. International Journal for Numerical Methods in Engineering, 72(9), pp.1111-1134.

[14] Wang, M., Feng, Y.T., Owen, D.R.J. and Qu, T.M., 2019. A novel algorithm of immersed moving boundary scheme for fluid-particle interactions in DEM-LBM. Computer Methods in Applied Mechanics and Engineering, 346, pp.109-125.

[15] Boutt, D.F., Cook, B.K., McPherson, B.J. and Williams, J.R., 2007. Direct simulation of fluid- solid mechanics in porous media using the discrete element and lattice- Boltzmann methods. Journal of Geophysical Research: Solid Earth, 112(B10).

[16] Wang, M., Feng, Y.T. and Wang, C.Y., 2017. Numerical investigation of initiation and propagation of hydraulic fracture using the coupled Bonded Particle-Lattice Boltzmann Method. Computers \& Structures, 181, pp.32-40.

[17] Wang, M., Feng, Y.T., Zhao, T.T. and Wang, Y., 2019. Modelling of sand production using a mesoscopic bonded particle lattice Boltzmann method. Engineering Computations, 36(2), pp.691-706.

[18] Cui, X., Li, J., Chan, A. and Chapman, D., 2012. A 2D DEM-LBM study on soil behaviour due to locally injected fluid. Particuology, 10(2), pp.242-252.

[19] Wang, M., Feng, Y.T., Pande, G.N., Chan, A.H.C. and Zuo, W.X., 2017. Numerical modelling of fluid-induced soil erosion in granular filters using a coupled bonded particle lattice Boltzmann method. Computers and Geotechnics, 82, pp.134-143.

[20] El Shamy, U. and Abdelhamid, Y., 2014. Modeling granular soils liquefaction using coupled lattice Boltzmann method and discrete element method. Soil Dynamics and Earthquake Engineering, 67, pp.119-132.

[21] Wang, M., Feng, Y.T., Wang, Y. and Zhao, T.T., 2017. Periodic boundary conditions of discrete element method-lattice Boltzmann method for fluid-particle coupling. Granular Matter, 19(3), p.43. 
[22] Cheng, H., Luding, S., Rivas, N., Harting, J. and Magnanimo, V., 2019. Hydro- micromechanical modeling of wave propagation in saturated granular crystals. International journal for numerical and analytical methods in geomechanics, 43(5), pp.1115-1139.

[23] Feng, Z. G. \& Michaelides, E. E. 2004. The immersed boundary-lattice Boltzmann method for solving fluidparticles interaction problems. Journal of Computational Physics, 195, 602-28.

[24] Wu, J. and Shu, C., 2009. Implicit velocity correction-based immersed boundary-lattice Boltzmann method and its applications. Journal of Computational Physics, 228(6), pp.1963-1979.

[25] Dash, S.M., Lee, T.S., Lim, T.T. and Huang, H., 2014. A flexible forcing three dimension IB-LBM scheme for flow past stationary and moving spheres. Computers \& Fluids, 95, pp.159-170.

[26] Noble, D.R. and Torczynski, J.R., 1998. A lattice-Boltzmann method for partially saturated computational cells. International Journal of Modern Physics C, 9(08), pp.1189-1201.

[27] Lallemand, P. \& Luo, L.-S. 2003. Lattice Boltzmann method for moving boundaries. Journal of Computational Physics, 184, 406-421.

[28] Wang, D., Leonardi, C.R. and Aminossadati, S.M., 2018. Improved coupling of time integration and hydrodynamic interaction in particle suspensions using the lattice Boltzmann and discrete element methods. Computers \& Mathematics with Applications, 75(7), pp.2593-2606.

[29] Peskin, C. S. 1977. Numerical analysis of blood flow in the heart. Journal of Computational Physics, 25, 220252.

[30] Zhang, H., Trias, F.X., Oliva, A., Yang, D., Tan, Y., Shu, S. and Sheng, Y., 2015. PIBM: Particulate immersed boundary method for fluid-particle interaction problems. Powder technology, 272, pp.1-13.

[31] Niu, X.D., Shu, C., Chew, Y.T. and Peng, Y., 2006. A momentum exchange-based immersed boundary-lattice Boltzmann method for simulating incompressible viscous flows. Physics Letters A, 354(3), pp.173-182.

[32] Uhlmann, M., 2005. An immersed boundary method with direct forcing for the simulation of particulate flows. Journal of Computational Physics, 209(2), pp.448-476.

[33] Wang, Z., Fan, J. and Luo, K., 2008. Combined multi-direct forcing and immersed boundary method for simulating flows with moving particles. International Journal of Multiphase Flow, 34(3), pp.283-302.

[34] Wang, M., Feng, Y.T. and Wang, C.Y., 2016. Coupled bonded particle and lattice Boltzmann method for modelling fluid-solid interaction. International Journal for Numerical and Analytical Methods in Geomechanics, 40(10), pp.1383-1401.

[35] Qian, Y.H., d'Humières, D. and Lallemand, P., 1992. Lattice BGK models for Navier-Stokes equation. Europhysics Letters, 17(6), p.479.

[36] Ergun, S., 1952. Fluid flow through packed columns. Chem. Eng. Prog., 48, pp.89-94. 
[37] Whitaker, S., 1986. Flow in porous media I: A theoretical derivation of Darcy's law. Transport in porous media, 1(1), pp.3-25.

[38] Han, Y. and Cundall, P.A., 2013. LBM-DEM modeling of fluid-solid interaction in porous media. International Journal for Numerical and Analytical Methods in Geomechanics, 37(10), pp.1391-1407.

[39] Wang, M., Feng, Y.T., Pande, G.N. and Zhao, T.T., 2018. A coupled 3- dimensional bonded discrete element and lattice Boltzmann method for fluid- solid coupling in cohesive geomaterials. International Journal for Numerical and Analytical Methods in Geomechanics, 42(12), pp.1405-1424.

[40] He, X., Zou, Q., Luo, L.S. and Dembo, M., 1997. Analytic solutions of simple flows and analysis of nonslip boundary conditions for the lattice Boltzmann BGK model. Journal of Statistical Physics, 87(1-2), pp.115-136.

[41] d'Humieres, D., 2002. Multiple-relaxation-time lattice Boltzmann models in three dimensions. Philosophical Transactions of the Royal Society of London. Series A: Mathematical, Physical and Engineering Sciences, 360(1792), pp.437-451.

[42] Guo, X., Zhong, C., Zhuo, C. and Cao, J., 2014. Multiple-relaxation-time lattice Boltzmann method for study of two-lid-driven cavity flow solution multiplicity. Theoretical and Computational Fluid Dynamics, 28(2), pp.215-231.

[43] Ladd, A.J., 1994. Numerical simulations of particulate suspensions via a discretized Boltzmann equation. Part 1. Theoretical foundation. Journal of fluid mechanics, 271, pp.285-309.

[44] Ladd, A.J.C. and Verberg, R., 2001. Lattice-Boltzmann simulations of particle-fluid suspensions. Journal of statistical physics, 104(5-6), pp.1191-1251.

[45] Mei, R., Luo, L.S. and Shyy, W., 1999. An accurate curved boundary treatment in the lattice Boltzmann method. Journal of computational physics, 155(2), pp.307-330.

[46] Maier, R.S., Bernard, R.S. and Grunau, D.W., 1996. Boundary conditions for the lattice Boltzmann method. Physics of Fluids, 8(7), pp.1788-1801.

[47] Wan D, Turek S. 2006. Direct numerical simulation of particulate flow via multigrid FEM techniques and the fictitious boundary method. Int J Numer Methods Fluids, 51(5):531-566. 